\title{
In-Situ Calibration of KM3NeT
}

\author{
Karel Melis* \\ Nikhef Institute for Subatomic Physics, Amsterdam, The Netherlands \\ E-mail: kmelis@nikhef.nl
}

\section{On behalf of the KM3NeT Collaboration}

The KM3NeT detector is a Čherenkov neutrino telescope being installed at two sites in the Mediterranean deep-sea. Its infrastructure will comprise several thousands of multi-PMT digital optical modules arranged in three-dimensional spatial arrays. The first two detection lines of KM3NeT are taking data since December 2015 and May 2016 respectively. These lines are the first of 2x115 KM3NeT/ARCA strings optimized for the detection of high-energy neutrino sources. Each line is about $700 \mathrm{~m}$ high and hosts 18 DOMs equally spaced by $36 \mathrm{~m}$. Results of the first detection lines are presented, including in-situ calibration and validation of the nanosecond timing capabilities, comparison with data from optical flashers, and the reconstruction of the first atmospheric muons. The results highlight the excellent photon detection capabilities of the KM3NeT multi-PMT technology and signify an important verification of the design.

35th International Cosmic Ray Conference

10-20 July, 2017

Bexco, Busan, Korea

${ }^{*}$ Speaker. 


\section{Introduction}

The KM3NeT detectors are currently under construction in the Mediterranean deep sea to 1) determine fundamental neutrino properties by measuring the atmospheric neutrino flux with the KM3NeT/ORCA detector and 2) identify and study the sources of high-energy cosmic neutrinos using the KM3NeT/ARCA detector. The properties of neutrino induced events, most notably the neutrino flavour, direction and energy, are reconstructed from the time-dependent pattern of detected photons as registered by the three-dimensional grid of photomultiplier tubes (PMTs). In order to achieve the excellent reconstruction resolutions shown and discussed in [1], [2] and [3], a good understanding and calibration of the PMT properties is of crucial importance.

The main objectives of the KM3NeT in-situ calibration are 1) to determine the relative PMT efficiencies and PMT-specific transit times ('intra-DOM calibration') and 2) to measure the DOMspecific optical fiber lenghts ('inter-DOM calibration'). By design, the time and efficiency calibration of the detector is done after deployment in the sea using the optical backgrounds from ${ }^{40} \mathrm{~K}$ decays and atmospheric muons, allowing for constant monitoring with high statistics, independent of changes in the detector settings such as the PMT high voltage settings.

In these proceedings results of the first two KM3NeT Detection Units will be presented. A data-taking period of 228 hours from December $23^{\text {th }} 2016$ until January the $2^{\text {nd }} 2017$ has been considered, with a recorded total lifetime of 227 hours and 42 minutes.

\section{The KM3NeT Detector}

KM3NeT's base detection unit is the custom designed Digital Optical Module (DOM). It comprises a pressure-resistant glass sphere containing 31 outward looking PMTs with accompanying electronics. Eighteen DOMs are attached to two vertical Dyneema ${ }^{\circledR}$ ropes, kept upright by a buoy and attached to an anchor. Such a set is called a detection unit (DU); 115 DUs are grouped together to form a so-called building block. The hits on the 31 PMTs in each DOM are time-stamped and sent to shore for further processing using a network of optical fibers and power cables.

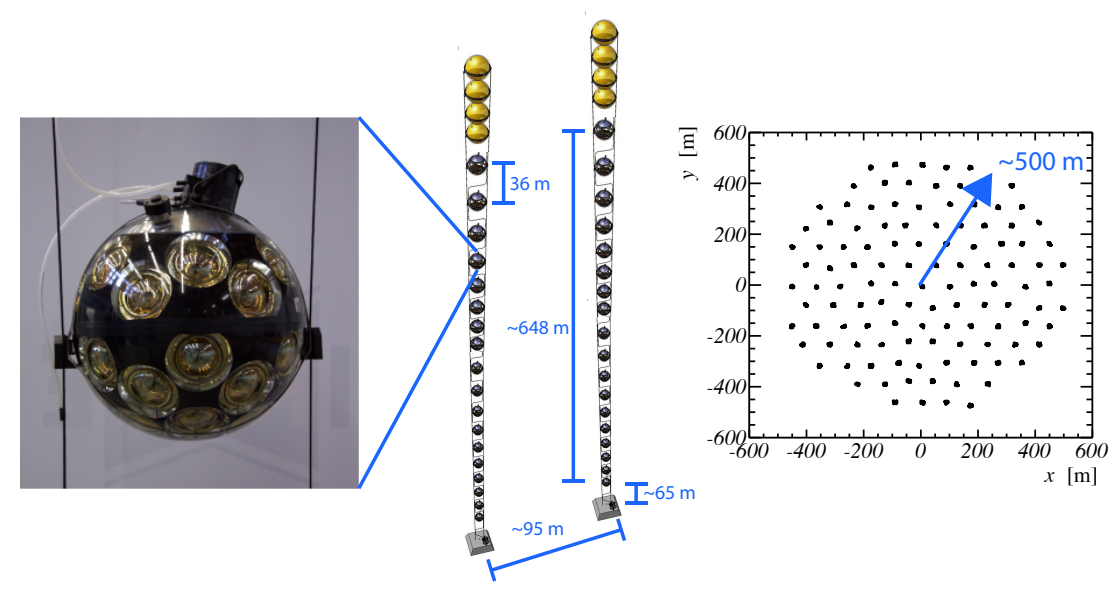

Figure 1: Schematic outline of a KM3NeT/ARCA building block. 
Currently, three building blocks are foreseen; two KM3NeT/ARCA building blocks and one KM3NeT/ORCA block to be installed offshore Sicily (Italy) and Toulon (France) respectively. For the detection of high-energy cosmic neutrinos the instrumented volume of the ARCA building block will be approximately $0.5 \mathrm{~km}^{3}$ with a vertical DOM spacing of approximately 36 meters and a horizontal DU spacing of about 95 meters (figure 1). The denser DOM spacing of the ORCA building block is optimal for the measurement of the neutrino mass hierarchy [1].

In order to cross-check the inter-DOM time calibration with the procedure described in these proceedings, each DOM is equiped with a LED ('Nanobeacon') capable of illuminating the surrounding DOMs up to a distance of approximately 400 meters. In the future, dedicated stand-alone calibration units equipped with laser beacons are foreseen. More details on the KM3NeT infrastructure can be found in [1] and [3].

\section{Intra-DOM Calibration using ${ }^{40} \mathrm{~K}$ Decays}

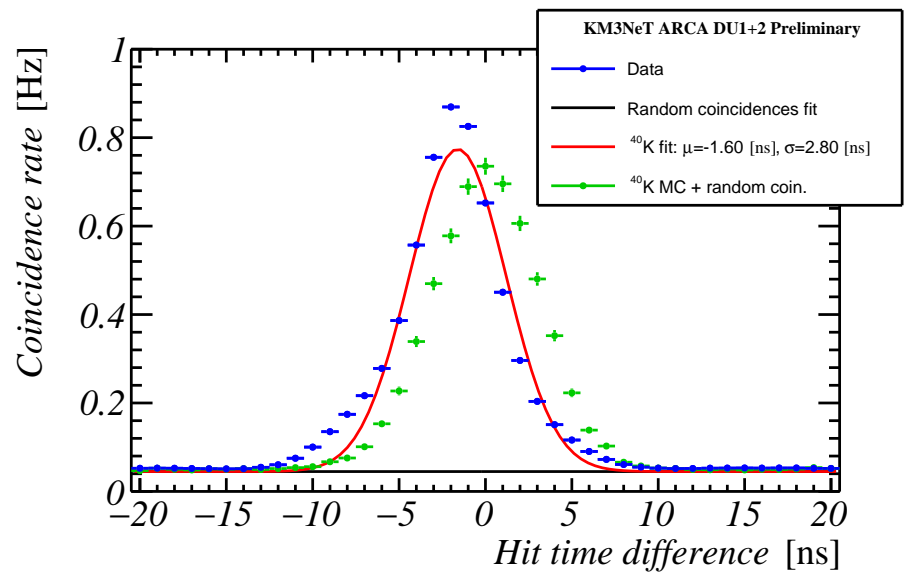

Figure 2: Coincidence rate as function of the hit time difference for an arbitrarily chosen pair of adjacent PMTs observed in data without calibration (blue points), with fit as described in the text (red line). The background rate estimated from the tail of the distribution in data (black solid line) is added to the simulated hit time difference distribution (green points).

The vast majority of coincident hits on $\geq 2$ PMTs within a DOM in a time window of $25 \mathrm{~ns}$ (called 'L1 hits') are caused by ${ }^{40} \mathrm{~K}$ decays in the vicinity of the DOM. The distribution of the hit time difference $(\Delta t)$ between these coincident hits is expected to be approximately gaussian distributed, where the mean is the difference between the transit times $\left(t_{i}^{0}\right)$ of the involved PMTs, the width is related to the PMT transit time spreads $\left(T T S_{i}\right)$ and the integral scales with the product of the PMT efficiencies $E_{i}$ :

$$
\begin{aligned}
R_{i, j}(\Delta t) & =R\left(\theta_{i, j}\right) \cdot E_{i} \cdot E_{j} \cdot \frac{1}{\sqrt{2 \pi} \sigma_{i, j}} \exp \left[\frac{\left(\Delta t-t_{i}^{0}+t_{j}^{0}\right)^{2}}{2 \sigma_{i, j}^{2}}\right]+R_{i, j}^{\text {random }} \\
\sigma_{i, j}^{2} & =T T S_{i}^{2}+T T S_{j}^{2}+\sigma_{40 K}^{2}
\end{aligned}
$$


In this the parametrization of the expected total coincidence rate of a PMT pair $R\left(\theta_{i, j}\right)$ and intrinsic time spread due to the spatial distribution of the ${ }^{40} \mathrm{~K}$ decays $\left(\sigma_{40 K}\right)$ are determined from dedicated Monte Carlo simulations with the KM3NeT software package OMGsim [4], which uses Geant4 [5] and includes a detailed (optical) model of the KM3NeT DOM, thus facilitating a detailed photon propagation simulation.

From the simulated events, the intrinsic time spread from the spatial distribution of ${ }^{40} \mathrm{~K}$ decays is determined to be $\sigma_{40 K}=0.54 \mathrm{~ns}$. The (expected) total coincidence rate as function of the PMT angular separation is shown in figure 3. The expected total coincidence rate $R\left(\theta_{i, j}\right)$ is parametrised by an exponential with a polynomial argument:

$$
\begin{aligned}
R\left(\theta_{i, j}\right) & =\exp \left[p_{1}+c t_{i, j} *\left(p_{2}+c t_{i, j} *\left(p_{3}+c t_{i, j} * p_{4}\right)\right)\right] \\
c t_{i, j} & =\cos \left(\theta_{i, j}\right) \\
p_{1} & =-1.07061, p_{2}=3.17173, p_{3}=-1.35769, p_{4}=1.6885
\end{aligned}
$$

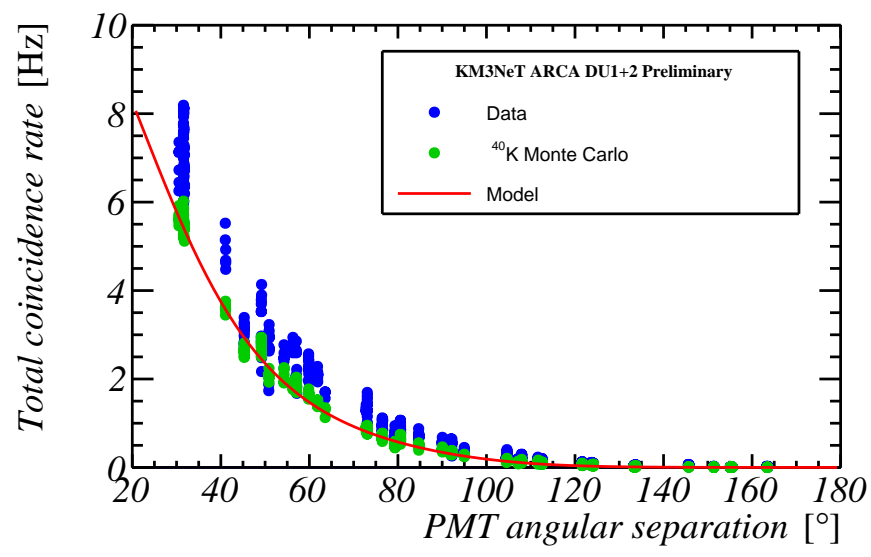

Figure 3: Total coincidence rate (random coincidence background subtracted) as function of the PMT angular separation in data (blue) and in simulations (green). The red line is a fit of the green points. More details in the text.

The flat background from random coincidences $R_{i, j}^{\text {random }}$ are determined by a fit of the tails of each distribution and subtracted from the $\Delta t$ distributions. After this, a simultaneous fit to the hit time distributions of all $0.5 \cdot 31 \cdot 30=465$ PMT pairs in a DOM provides the 31 PMT time offsets, transit time spreads and PMT efficiencies.

PMT time offsets and transit time spreads In figure 4, the fitted PMT time offsets using the ${ }^{40} \mathrm{~K}$ calibration are cross-checked with the maxima of hit time distributions of nanobeacon light seen on a selection of PMTs in the lower half of the DOM one floor higher. As can be seen, both methods are in very good agreement $(\sigma=0.7 \mathrm{~ns})$.

In addition to an accurate timing calibration, a small PMT transit time spread is advantageous for the event reconstruction algorithms. Using the fit described, the transit time spreads of the three inch PMTs used in KM3NeT average at a value of $2.07 \mathrm{~ns}$. 

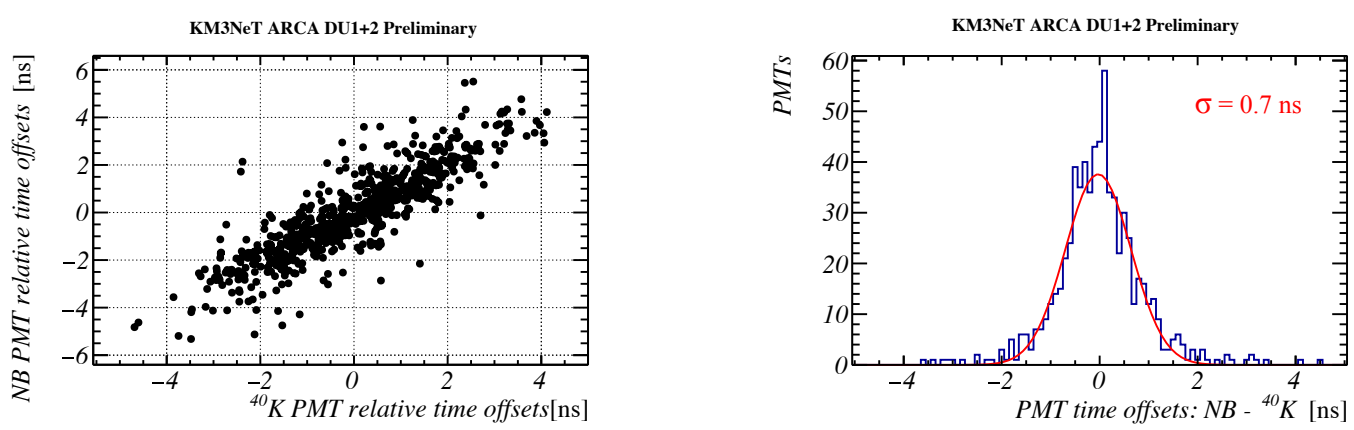

Figure 4: PMT relative time offsets obtained using an analysis of the LED nanobeacon data in comparison with those obtained using the ${ }^{40} \mathrm{~K}$ intra-DOM time calibration. The averages of all fitted PMT time offsets within each DOM are set to zero.

PMT relative efficiencies The ${ }^{40} \mathrm{~K}$ coincidence rates seen in data exceed those expected from the simulations (figure 3), indicating that the PMTs have higher efficiencies than the assumed nominal PMT model used in the simulations. This can also be seen in the distribution of the fitted PMT efficiencies shown in figure 5, and is consistent with earlier measurements from the PPM-DU prototype [8]. A more detailed study shows that the PMTs with low efficiencies are located close to the support structure of the DOM (figure 1), most notably the two attachments to the Dyneema ${ }^{\circledR}$ ropes.

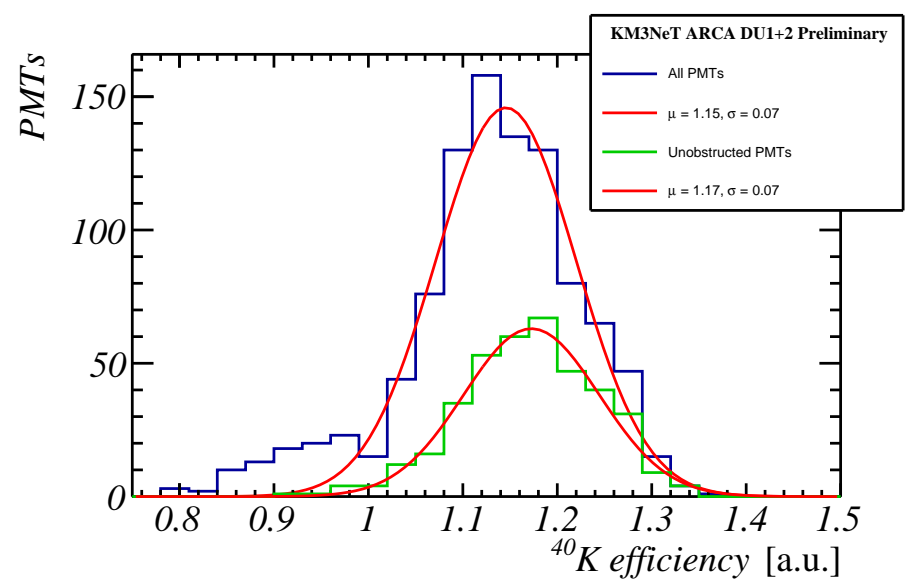

Figure 5: Distribution of fitted PMT efficiencies for all PMTs (blue) and for only PMTs with a free field of view, i.e. excluding all PMTs close to the surrounding support structure (green). The red lines are the results of gaussian fits to both distributions.

\section{Inter-DOM Time Calibration using Atmospheric Muon Tracks}

The intra-DOM calibration only constrains the difference between the PMT transit times 
within a DOM. For the determination of the inter-DOM time offsets, a method has been developed using reconstructed atmospheric muon tracks.

All events with at least 7 DOMs registering an L1 hit are reconstructed with the default KM3NeT track reconstruction algorithm [2], iteratively excluding one of the hit DOMs from the track fit. The distribution of the time difference between the measured hit time on the excluded DOM and the expectation from the reconstructed track (referred to as the hit time residual distribution) is compared to the distribution obtained by applying the same procedure to Monte Carlo simulated events (figure 6).

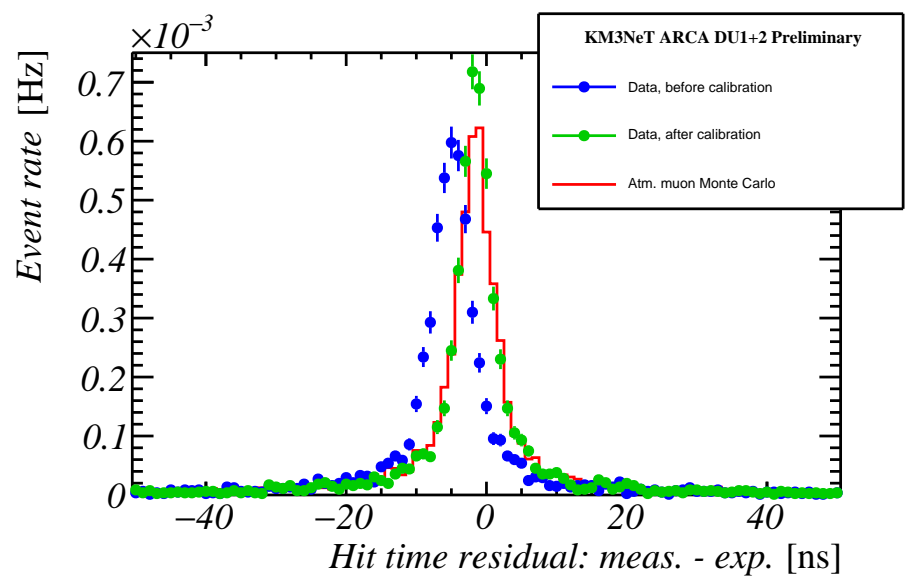

Figure 6: Hit time residual distributions obtained from Monte Carlo simulations (red) and data before (blue) and after (green) DOM time offset calibration.

The set of Monte Carlo simulated events has been produced on a run-by-run basis, including a detailed model of the PMTs using the fitted PMT efficiencies determined with the ${ }^{40} \mathrm{~K}$ intra-DOM calibration method. The atmospheric muon flux, photon propagation through the sea water and detector response are simulated using the mupage, $\mathrm{km} 3$ and JPP software packages respectively [6]. These simulations have also been used in e.g. [7].

The fact that the hit time residual distribution before calibration (the blue points in figure 6) does not peak at zero indicates that the DOM time offset is not yet calibrated. The best fitting DOM time offset is obtained by maximizing the agreement between the data and the Monte Carlo model:

$$
Q(\Delta t)=\prod_{b i n s}\left[\operatorname{Poisson}\left(\text { data }_{i} \mid M C_{i}\right)\right]
$$

Since the reconstructed track parameters are correlated with the DOM time offsets, the procedure outlined above is repeated until convergence is achieved. The result after convergence is given by the green distribution in figure 6 . As can be seen, the agreement between data and Monte Carlo is convincing, both in shape and in overall rate.

\section{Data Monte-Carlo Comparison}

A detailed measurement of the agreement between data, ${ }^{40} \mathrm{~K}$ Monte Carlo and atmospheric muon Monte Carlo is provided by the rate of PMTs hit in coincidence (figure 7). The coincidence 
rate ranges over eight orders of magnitude from a regime dominated by ${ }^{40} \mathrm{~K}$ decays (PMT multiplicities $\leq 7$ ) to a regime dominated by coincidences from atmospheric muons. As can be seen, data and Monte Carlo agree well over the entire range, underlining the good understanding of the KM3NeT detector and it's PMTs.

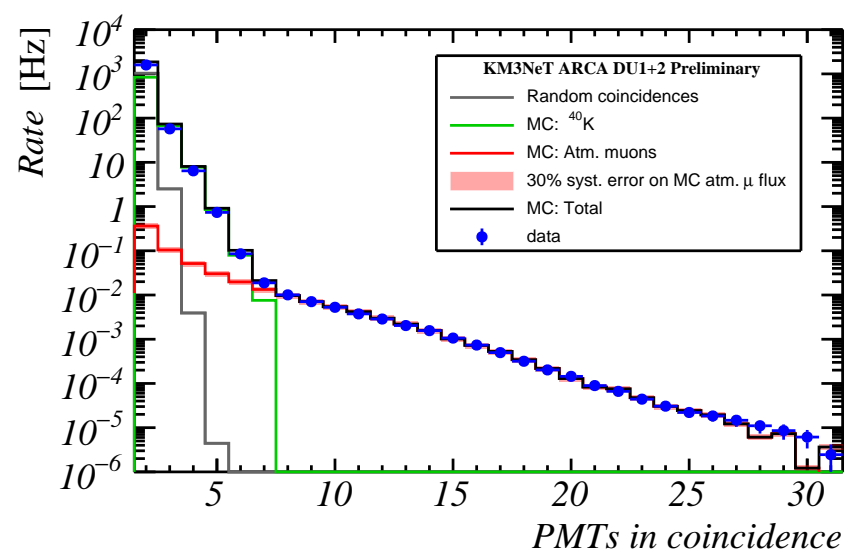

Figure 7: Rate of coincident hits in a non-sliding time window of 25 nanoseconds as function of the number of hit PMTs for data (blue) and simulations (black). The total coincidence rate from simulations is given by the sum of random coincidences of the PMT singles rates of $6.7 \mathrm{kHz}$ (gray), genuine ${ }^{40} \mathrm{~K}$ coincidences (green), atmospheric muons (red) and combinations of these. The PMT efficiencies in the ${ }^{40} \mathrm{~K}$ simulation are varied within the systematic uncertainties in order to match the slope observed in data. The systematic uncertainty in the overall atmospheric muon flux of $30 \%$ is given by the red band.

\section{References}

[1] S. Adrían-Martínez et al. (KM3NeT Collaboration), Letter of Intent for ARCA and ORCA, J. Phys. G: Nucl. Part. Phys. 43 (2016), 084001.

[2] K. Melis, A. Heijboer, M. de Jong, KM3NeT/ARCA Event Reconstruction Algorithms in these proceedings.

[3] A. Heijboer, KM3NeT, Physics and status in these proceedings.

[4] M. Colomer, D. Dornic, V. Kulikovskiy, Detailed KM3NeT optical module simulation with Geant4 and supernova neutrino detection study in these proceedings.

[5] geant4.web.cern.ch

[6] A. Margiotta, Common simulation tools for large volume neutrino detectors, Nucl. Instrum. Meth. 98 (2013), A725.

[7] M. Jongen, Detailed KM3NeT optical module simulation with Geant4 and supernova neutrino detection study in these proceedings.

[8] S. Adrían-Martínez et al. (KM3NeT Collaboration), The prototype detection unit of the KM3NeT detector, The European Physical Journal C 76 (2016), 1434-6052 